\title{
Simulated random surfaces and effective string models in 3D Z(2) gauge theory
}

\section{Tomasz Korzec}

Humboldt Universitat zu Berlin, Institut fur Physik, Newtonstrasse 15, D-12489 Berlin, Germany

E-mail: korzec@physik.hu-berlin.de

\section{Ulli Wolff*}

Humboldt Universitat zu Berlin, Institut fur Physik, Newtonstrasse 15, D-12489 Berlin, Germany

E-mail: uwolff@physik.hu-berlin.de

\begin{abstract}
All-order strong coupling simulations have been used to derive precise energies of string states in the confined phase of three dimensional Z(2) lattice gauge theory. The behavior of the ground state energy is here compared with predictions of effective string theory. Our new data reported here are consistent with known universal terms of the long string length $\left(L_{0}\right)$ expansion known from effective string models in the continuum limit. Our precision is however still not sufficient to disentangle non-univeral terms of order $L_{0}^{-7}$.
\end{abstract}

31st International Symposium on Lattice Field Theory LATTICE 2013

July 29 - August 3, 2013

Mainz, Germany

\footnotetext{
*Speaker.
} 


\section{Introduction}

Starting from the work of Prokof'ev and Svistunov [1] a number of scalar lattice field theories have been simulated by so-called worm algorithms. An overview and more references can be found in [2]. The main ingredient of the method is the replacement of the original path integral by an untruncated strong coupling series for the partition function. Contributions are represented by an ensemble of positively weighted graphs. For any finite lattice these series are convergent for all values of the bare parameters and may thus be considered as equivalent to the original systems. To realize the convergence for interesting areas in parameter space, high orders are important and the summation is only possible by stochastic methods like Monte Carlo simulation. The observation in [1] has been that in such simulations it is highly profitable to enlarge the graph space from the graphs of the partition function to those required for the fundamental two point correlation between arbitrary pairs of sites $u, v$, the 'head' and 'tail' of the worm. In the graphical language this introduces a pair of point defects where lines can end that otherwise have to form closed loops. Although the relevant graphs are essentially sampled by local deformations only, very little critical slowing down hampers the computation of relevant observables. Moreover, by sampling the enlarged graph space, a very efficient computation of the two point function and quantities like the mass gap becomes possible. As an additional bonus it is shown in [3] that the exponential decay of such correlations can be numerically followed to large separations without the usual degradation of the signal to noise ratio. This is achieved by introducing suitable weight factors into the graph ensemble that is simulated. A further advantage of worm methods is that in some cases, in particular with nonzero chemical potential, a sign problem present in the original formulation is absent for the equivalent graphs and is hence cured.

The success story outlined above has so far mainly been restricted to nonlinear sigma models of the $\mathrm{O}(N)$ and $\mathrm{CP}(N-1)$ type as prototype scalar field theories. Even setting aside fermions a natural next candidate to study are gauge theories. For Abelian theories $(\mathrm{Z}(N), \mathrm{U}(1))$ the generalization to the strong coupling representation of the partition function is at least superficially straight forward. Admissible defects are now superpositions of arbitrary closed loops instead of point defects in the scalar models. The loop defects are related to Wilson loop observables in a similar way as the point pairs connect to scalar correlations. A key difference is now that possible loop defects of arbitrary shape form a very much larger set than the possible locations of pairs of points. In our recent article [4] we have studied $\mathrm{Z}(2)$ gauge theory ${ }^{1}$ in three dimensions, which is dual to the Ising spin (or $\mathrm{O}(1)$ sigma) model. In a series of simulations at the critical point we have experimented with various subsets of such line defects but have failed to identify a set that is efficient with regard to slowing down. We have then changed our direction: we have accepted critical exponents around two but tailored the simulated ensemble to optimize the estimation of Polyakov line two point functions. Here we have indeed succeeded in achieving a separation independent signal to noise ratio which allows for the very precise calculation of the ground state 'quark-antiquark' potential and the properties of string states in a finite volume. In the rest of this contribution we discuss these results and their matching with effective string theory predictions. Further details on the algorithmic aspects of our investigation are summarized, including graphical illustrations, in the contribution by Tomasz Korzec [6].

\footnotetext{
${ }^{1}$ For earlier related attempts in U(1) gauge theory there are more references in [4], in particular [5].
} 


\section{Polyakov line correlation function}

Our calculation takes place in Z(2) gauge theory on a periodic lattice of size $L_{0} \times L \times L$. Employing the standard Wilson action defined on plaquettes the partition function is given by

$$
Z=\sum_{\left\{\sigma_{\mu}(x)= \pm 1\right\}} \mathrm{e}^{\beta \sum_{x, \mu<v} \sigma_{\mu v}(x)}
$$

where $\sigma_{\mu v}(x)$ is the product of the links $\sigma_{\mu}(x)$ around a plaquette. The Polyakov line correlation function is defined by

$$
G(\vec{x})=\langle\pi(\vec{x}) \pi(\overrightarrow{0})\rangle, \quad \pi(\vec{x})=\prod_{x_{0}=0}^{L_{0}-1} \sigma_{0}\left(x_{0}, \vec{x}\right) .
$$

In our random surface simulation there are straight line defects of length $L_{0}$ at two dimensional locations $\vec{u}$ and $\vec{v}$ which randomly wander over the lattice [4], [6]. The random surface ensemble is constructed such that we can prove the relation

$$
G(\vec{x})=\rho(\vec{x}) \frac{\left\langle\left\langle\delta_{\vec{x}, \vec{u}-\vec{v}}\right\rangle\right\rangle}{\left\langle\left\langle\delta_{\vec{u}, \vec{v}}\right\rangle\right\rangle} .
$$

The function $\rho(\vec{x})$ has been incorporated to bias our updates and is exactly canceled here to yield $G(\vec{x})$. It has been modeled to capture much of the variation of $G$ with $|\vec{x}|$ and this is what leads to our favorable signal to noise behavior, because the Monte Carlo only computes the slowly varying correction factor that turns $\rho$ into the exact correlation.

On the theoretical side $G(\vec{x})$ can be viewed as the partition functions of the $\mathrm{Z}(2)$ gauge theory with static charges separated by $\vec{x}$. If we employ the transfer matrix with respect to the temporal direction we arrive at the formula [7]

$$
G(\vec{x})=\sum_{n \geqslant 0} w_{n} \mathrm{e}^{-V_{n}(\vec{x}) L_{0}}
$$

where $w_{n}$ are integer multiplicity factors. Assuming a gap we note that in the limit of large $L_{0}$ the sum can be approximated by the groundstate potential term $n=0$.

We may however analyze the same correlation also in terms of the transfer matrix in one of the two spatial directions, say $\mu=1$. Projecting to zero momentum in the $\mu=2$ direction we arrive at

$$
\sum_{x_{2}=0}^{L-1} G\left(x_{1}, x_{2}\right)=\sum_{n \geqslant 0}\left|v_{n}\right|^{2} \mathrm{e}^{-\tilde{E}_{n} x_{1}}
$$

Here $v_{n}$ are non-trivial matrix elements and we have assumed $x_{1} \ll L, \tilde{E}_{n} L \gg 1$ to have purely exponential decay. The energy levels $\tilde{E}_{n}$ refer to flux states created by the Polyakov line operator and are thus expected to be asymptotically proportional to its length $L_{0}$.

Moreover, in [7] Lüscher and Weisz have shown that the string state energies contain enough information to reconstruct the complete correlator

$$
G(\vec{x})=\sum_{n \geqslant 0}\left|v_{n}\right|^{2} 2 r\left(\frac{\tilde{E}_{n}}{2 \pi r}\right) K_{0}\left(\tilde{E}_{n} r\right) \quad(r=|\vec{x}|) .
$$

As one may expect form the presence of $r$ this formula holds in the continuum theory and $K_{0}$ denotes a Bessel function. As $K_{0}$ decays exponentially for large arguments, in particular the ground state energy $\tilde{E}_{0}$ controls the large distance fall-off of $G(\vec{x})$ with an asymptotic area law. 


\section{Effective string theory}

In effective string theories the fundamental degrees of freedom consist of a two dimensional surface - the time evolution of the string - embedded in $D \geqslant 3$ dimensional space. It furnishes an approximate description of large Wilson loops (or Polyakov loop pairs) in gauge theories with the loop position entering as the locus of Dirichlet boundary conditions for the edge of the surface. The functional integral over all surface configurations with a general action respecting the usual symmetries leads to a non-renormalizable theory [7] that is in some sense similar to chiral perturbation theory. It predicts for instance an expansion of the lowest potentials $V_{n}$ in (2.4) in powers of $r^{-1}$. In each order only a finite number of free parameters of the general action enter. In the closed string interpretation expansions of the lowest $\tilde{E}_{n}$ in powers of $L_{0}^{-1}$ can be derived.

It turns out that for $D=3$ the following result holds for the ground state energy,

$$
\tilde{E}_{0}=\sigma L_{0}-\frac{\pi}{6 L_{0}}-\frac{\pi^{2}}{72 \sigma L_{0}^{3}}-\frac{\pi^{3}}{432 \sigma^{2} L_{0}^{5}}+\mathrm{O}\left(L_{0}^{-7}\right) .
$$

Note that to this order the string tension $\sigma$ is the only dimensionful parameter that enters. The $L_{0}^{-5}$ has been shown to be parameter free ('universal') (for $D=3$ ) in [8] while for the earlier terms this result was already given in [7]. Note that the $L_{0}^{-1}$ term (one loop in string theory) is the so-called Lüscher term that has been known for a long time [9], [10]. We note that also the absence of even inverse powers seems to be a nontrivial result.

In [11] the Nambu-Goto geometric string action is used instead of a general effective action restricted by symmetries only. This leads to the formal all-order result

$$
z^{2}=s^{2}\left(1-\frac{1}{3 s}\right), \quad s=\frac{\sigma L_{0}^{2}}{\pi}, \quad z=\frac{\tilde{E}_{0} L_{0}}{\pi}, \quad s, z \rightarrow \infty
$$

which upon expansion implies the terms exhibited explicitly in (3.1). Due to inconsistencies in the Nambu-Goto theory, the higher orders are not expected to be correct, but at some point free coefficients that can distinguish between different gauge models are expected to appear.

\section{Fitting string theory to new data}

In [4] we have given results for $\tilde{E}_{0}$ for $\mathrm{Z}(2)$ gauge theory at one lattice spacing with the string tension in lattice units $\sigma \cong 0.0044, L=64$, and $L_{0}=6 \ldots 28$. To have better control over the continuum limit we here report on additional data for an approximately two times smaller lattice spacing at $\beta=0.75146$. They are collected in table 1 . An iteration of the surface algorithm applied is detailed in [4] and corresponds to an order of Volume CPU effort comparable to a sweep of a local algorithm. Again, as for the first series of measurements, we found long plateaus for effective masses $\tilde{E}_{0}$ and the extraction of numerical values proceeded as before.

The Nambu Goto result (3.2) would imply that the data can be fitted by only two terms

$$
\frac{\tilde{E}_{0}^{2}}{L_{0}^{2}}=\sigma^{2}+c_{1} \frac{1}{L_{0}^{2}}
$$




\begin{tabular}{|l|l|l|l|l|l|}
\hline$L_{0}$ & $\tilde{E}_{0}$ & stat & $L_{0}$ & $\tilde{E}_{0}$ & stat \\
\hline 12 & $0.080998(6)$ & 85 & 36 & $0.381209(55)$ & 69 \\
\hline 16 & $0.139831(9)$ & 74 & 40 & $0.426695(82)$ & 58 \\
\hline 20 & $0.192035(20)$ & 33 & 44 & $0.47173(11)$ & 57 \\
\hline 24 & $0.241174(30)$ & 33 & 48 & $0.51682(14)$ & 54 \\
\hline 28 & $0.288694(43)$ & 32 & 52 & $0.56187(18)$ & 52 \\
\hline 32 & $0.335173(39)$ & 72 & 56 & $0.60661(24)$ & 48 \\
\hline
\end{tabular}

Table 1: New data complementing those listed in [4]. Parameters are $\beta=0.75146, L=128$ and the statistics is given as multiples of $10^{6}$ iterations.

\begin{tabular}{|l|l|l|l|}
\hline$L_{0, \min }$ & $\chi^{2} / \mathrm{dgf}$ & $\sigma$ & $r$ \\
\hline 24 & $6.5 / 7$ & $0.0109986(15)$ & $0.9986(41)$ \\
\hline 20 & $6.5 / 8$ & $0.0109986(10)$ & $0.9987(20)$ \\
\hline 16 & $110 / 9$ & & \\
\hline
\end{tabular}

Table 2: Resulting fit parameters (4.1), (4.2) when including $L_{0} \geqslant L_{0, \min }$.

where in addition it even fixes $c_{1}$ by

$$
r=\left(\frac{3 c_{1}}{\pi \sigma}\right)^{2}=1
$$

We perform such a fit with a free value $c_{1}$ and, to have visible error bars at all, plot immediately the deviation from the fit in figure 1. In table 2 we give the achieved $\chi^{2}$ values depending on how small $L_{0}$ we include in the fit. We see completely acceptable and stable fits under varying $L_{0, \min }=20,24$, but find that there is no way to include $L_{0}=16$. The resulting values of $r$ are compatible with one, and hence at this lattice spacing the Nambu Goto form (3.2) is consistent with our data for $L_{0} \geqslant 20 a$. This is in contrast to the lattices in [4] which are physically matched with just a coarser lattice spacing. There $r$ had a significant $1 \%$ deviation from one, whose interpretation as a lattice artefact is hence confirmed.

\section{Conclusions}

We have used our all-oder strong coupling, or random surface simulation method, to investigate the energy of string states at various length that is closely related to the large distance behavior of the potential in three dimensional $Z(2)$ gauge theory. The data fit well with the prediction of the Nambu Goto effective string theory. It turns out, however, that our fit is sensitive to only those terms in the prediction that are universal to all effective string actions with the right symmetries. This is seen if we compare our typical error $\delta \tilde{E}_{0} \approx 2 \times 10^{-5}$ with the size of the first (presumably) non-universal term implied by (3.2) that reads $5 \pi^{4} /\left(10368 \sigma^{3} L_{0}^{7}\right) \approx 10^{-6}$, both for $L_{0}=20$. It is the previous term that is borderline relevant by making a contribution of about the same size as $\delta \tilde{E}_{0}$. The $L_{0}^{-3}$ term is significantly tested by our result which also clearly and nicely supports the 


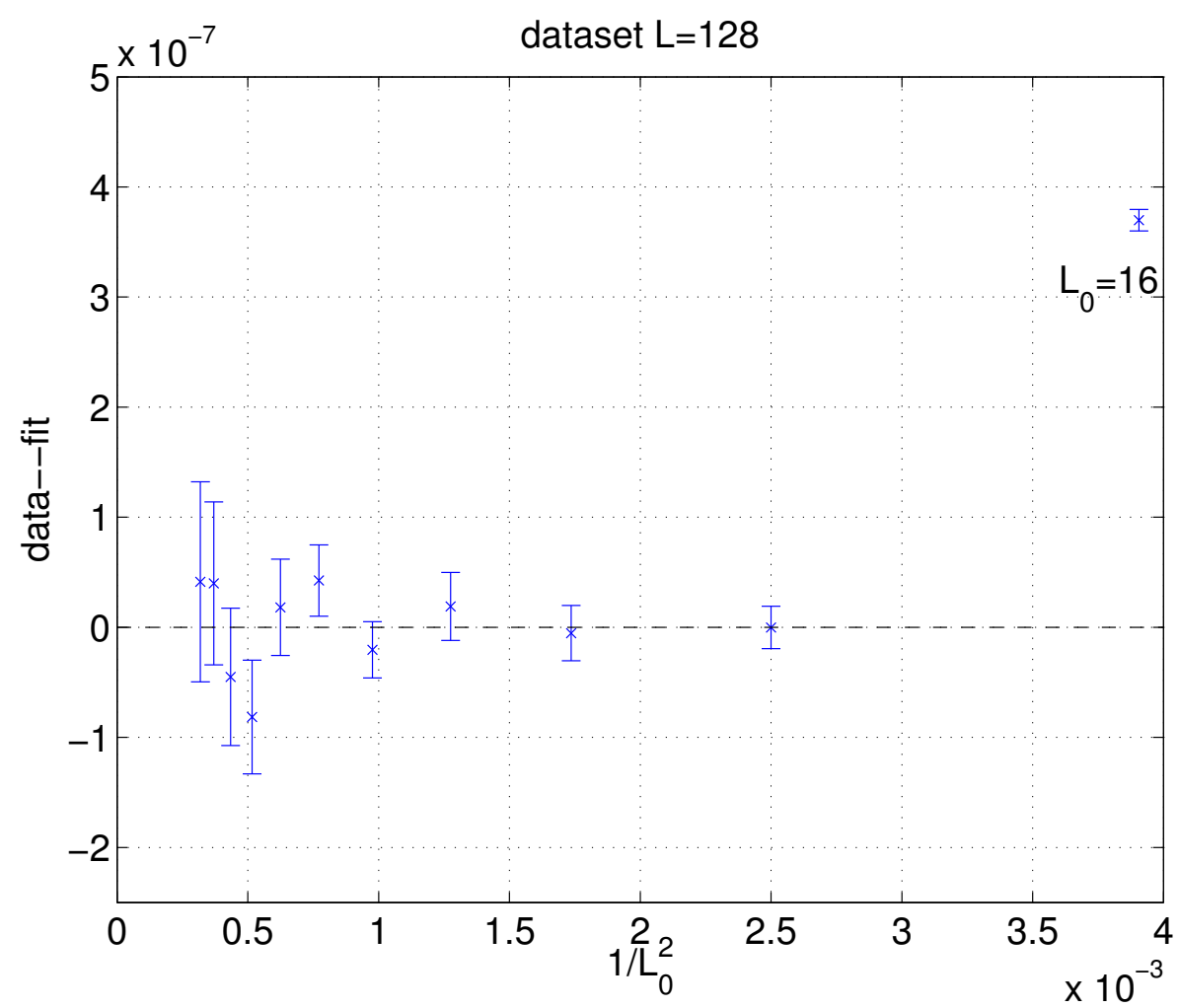

Figure 1: Difference between data and fit (4.1).

general behavior of the string theory asymptotics as well as the very abrupt break-away of the data from this form for smaller separations.

\section{References}

[1] N. Prokof'ev and B. Svistunov, Worm Algorithms for Classical Statistical Models, Phys. Rev. Lett. 87 (2001) 160601, [arXiv:0910.1393].

[2] U. Wolff, Strong coupling expansion Monte Carlo, PoS (LATTICE 2010) 020 , [arXiv:1009.0657].

[3] U. Wolff, Simulating the All-Order Strong Coupling Expansion I: Ising Model Demo, Nucl. Phys. B810 (2009) 491, [arXiv: 0808 .3934].

[4] T. Korzec and U. Wolff, Simulating the All-Order Strong Coupling Expansion V: Ising Gauge Theory, Nucl.Phys. B871 (2013) 145, [arXiv: 1212.2875].

[5] T. Korzec and U. Wolff, A worm-inspired algorithm for the simulation of Abelian gauge theories, POS (LATTICE 2010)029, [arXiv:1011.1359].

[6] T. Korzec, Simulating the Random Surface representation of Abelian Gauge Theories, POS (LATTICE 2013) 038.

[7] M. Lüscher and P. Weisz, String excitation energies in $S U(N)$ gauge theories beyond the free-string approximation, JHEP 0407 (2004) 014, [hep-th/ 0406205 ]. 
[8] O. Aharony and E. Karzbrun, On the effective action of confining strings, JHEP 0906 (2009) 012, [arXiv:0903.1927].

[9] M. Lüscher, K. Symanzik, and P. Weisz, Anomalies of the Free Loop Wave Equation in the WKB Approximation, Nucl.Phys. B173 (1980) 365.

[10] M. Lüscher, Symmetry Breaking Aspects of the Roughening Transition in Gauge Theories, Nucl.Phys. B180 (1981) 317.

[11] J. Arvis, The exact q anti-q potential in Nambu string theory, Phys.Lett. B127 (1983) 106. 